\title{
The Exact Degree of Precision of Generalized Gauss-Kronrod Integration Rules
}

\author{
By Philip Rabinowitz
}

\begin{abstract}
It is shown that the Kronrod extension to the $n$-point Gauss integration rule, with respect to the weight function $\left(1-x^{2}\right)^{\mu-1 / 2}, 0<\mu \leqslant 2, \mu \neq 1$, is of exact precision $3 n+1$ for $n$ even and $3 n+2$ for $n$ odd. Similarly, for the $(n+1)$-point Lobatto rule, with $-1 / 2<\mu \leqslant 1, \mu \neq 0$, the exact precision is $3 n$ for $n$ odd and $3 n+1$ for $n$ even.
\end{abstract}

1. Introduction. In this paper we shall consider the Kronrod extensions (KE) to the Gauss-Gegenbauer integration rules (GGIR) and the Lobatto-Gegenbauer rules (LGIR). The Gegenbauer polynomials, $C_{n}^{\mu}(x), \mu>-1 / 2$, are those polynomials which are orthogonal with respect to the weight function $w(x ; \mu) \equiv\left(1-x^{2}\right)^{\mu-1 / 2}$ and have the following normalization [4, p. 174]

$$
\int_{-1}^{1} w(x ; \mu) C_{n}^{\mu}(x) C_{m}^{\mu}(x) d x=\delta_{n m} h_{n \mu},
$$

where

$$
h_{n \mu}=\pi^{1 / 2} \Gamma(n+2 \mu) \Gamma(\mu+1 / 2) /((n+\mu) n ! \Gamma(\mu) \Gamma(2 \mu)),
$$

which implies that $C_{n}^{\mu}(x)=k_{n \mu} x^{n}+\ldots$, where

$$
k_{n \mu}=2^{n} \Gamma(n+\mu) /(n ! \Gamma(\mu)) \text {. }
$$

$C_{n}^{\mu}(x)$ is even (odd) if $n$ is even (odd). Special cases of $C_{n}^{\mu}(x)$, perhaps with a different normalization, are $T_{n}(x)$, the Chebyshev polynomials of the first kind $(\mu=0), P_{n}(x)$, the Legendre polynomials $(\mu=1 / 2)$, and $U_{n}(x)$, the Chebyshev polynomials of the second kind $(\mu=1)$.

The $n$-point GGIR is given by

$$
\text { If } \equiv \int_{-1}^{1} w(x ; \mu) f(x) d x=\sum_{i=1}^{n} w_{i} f\left(x_{i}\right)+c_{n \mu} M_{2 n}(f),
$$

where we have omitted the dependence of $w_{i}$ and $x_{i}$ on $\mu$ and $n, x_{i}$ are the zeros of $C_{n}^{\mu}(x)$,

$$
c_{n \mu}=2^{2 n} h_{n \mu} / k_{n \mu}^{2},
$$

and $M_{j}(f)$ is defined to be equal to $f^{(j)}(\xi) / 2^{j} j$ ! for some $\xi \in(-1,1)$. The corresponding LGIR has $n+1$ points and is given by

Received January 4, 1980.

AMS (MOS) subject classifications (1970). Primary 65D30.

Key words and phrases. Kronrod rule, Gauss integration rule, Lobatto integration rule, Gegenbauer polynomials, Szegö polynomials, Fourier coefficients. 


$$
I f=\sum_{i=1}^{n+1} \bar{w}_{i} f\left(\bar{x}_{i}\right)+\bar{c}_{n \mu} M_{2 n}(f),
$$

where the $\bar{x}_{i}$ are the zeros of $\left(1-x^{2}\right) C_{n-1}^{\mu+1}(x)$, and

$$
\bar{c}_{n \mu}=-\frac{2^{2 n} h_{n-1, \mu+1}}{k_{n-1, \mu+1}^{2}}=-4 c_{n-1, \mu+1} .
$$

Since the weights of the integration rules considered do not play a part in the discussion, we shall not treat them here except to remark that Monegato [9], [10] has shown that the weights $u_{i}$ in (8) below are positive for $0 \leqslant \mu \leqslant 1$ and the $v_{i}$, for $0 \leqslant \mu \leqslant 2$.

The KEGGIR is given by

$$
I f=\sum_{i=1}^{n} u_{i} f\left(x_{i}\right)+\sum_{i=1}^{n+1} v_{i} f\left(y_{i}\right)+E_{p_{n}}(f),
$$

where $E_{s}(f)=0$ if $f$ is a polynomial of degree $<s$ and $p_{n}=2[(3 n+3) / 2]$. The $y_{i}$ are the zeros of a certain polynomial $E_{n+1, \mu}(x)$ which we shall study in the next section. For the moment we state a result of Szego [16] that for $0 \leqslant \mu \leqslant 2$, the $y_{i}$ are real, lie in $[-1,1]$, and are separated by the $x_{i}$. (For $\mu \neq 0$, the $y_{i}$ lie in $(-1,1)$.) The corresponding KELGIR is given by

$$
\text { If }=\sum_{i=1}^{n+1} \bar{u}_{i} f\left(\bar{x}_{i}\right)+\sum_{i=1}^{n} \bar{v}_{i} f\left(\bar{y}_{i}\right)+E_{q_{n}}(f),
$$

where $q_{n}=2[(3 n+2) / 2]$, and the $\bar{y}_{i}$ are the zeros of $E_{n, \mu+1}(x)$. Thus, taking into account that $\mu>-1 / 2$, we see that practical KEGGIR's exist for $0 \leqslant \mu \leqslant 2$ and KELGIR's, for $-1 / 2<: l \leqslant 1$.

The first one to discover a KEGGIR was Kronrod [7] who dealt with the case $\mu=1 / 2$, the Gauss-Legendre or standard Gauss rule. Subsequently, Patterson [13], Piessens and Branders [14], and Monegato [11] improved on Kronrod's original work and extended his results to the usual Lobatto case $(\mu=1 / 2)$. Barrucand [2] was the first to point out the connection between the KE's and the Szego polynomials $E_{n+1, \mu}(x)$. KE's to other integration rules are discussed by Baratella [1], Kahaner and Monegato [5], Monegato [9], [12], and Ramskii [15].

In the entire literature on this subject, it is stated that the KE's have error terms which vanish for polynomials of degree less than $p_{n}$ (Gauss) or $q_{n}$ (Lobatto), and in Kronrod's tables, he gives the error in the integration of $x^{p_{n}}$ by the KEGGIR with $\mu=1 / 2$. However, nowhere is it proved that these KE's are of exact degree $p_{n}-1$ or $q_{n}-1$, as the case may be, that is, that there exists a polynomial of degree $p_{n}$ or $q_{n}$ for which the corresponding $\mathrm{KE}$ is not exact. Indeed, such a statement is not true for all $\mu$. Thus, as Monegato [9] points out, the KE of the $n$-point GGIR with $\mu=0$, the first Gauss-Chebyshev rule, is exact for polynomials of degree $\leqslant 4 n-1$ and in fact is identical with the KE of the corresponding $(n+1)$-point LGIR, being the $(2 n+1)$ point LGIR, the first Lobatto-Chebyshev rule. Furthermore, the KE of the $n$-point GGIR with $\mu=1$, the second Gauss-Chebyshev rule, is exact for polynomials of degree 
$\leqslant 4 n+1$ and, in fact, is identical with the $(2 n+1)$-point GGIR. In the present work, we shall show that, except for $\mu=0,1$ in the GGIR case and $\mu=0$ in the LGIR case, we have the result that the exact precision of the KEGGIR is $p_{n}-1$ while that of the KELGIR is $q_{n}-1$. Furthermore, if these rules are of simplex type, i.e., if we can express the error term in the form $K_{n \mu} f^{\left(p_{n}\right)}(\xi)$ or $K_{n \mu} f^{\left(q_{n}\right)}(\xi)$, which we have not been able to prove, then we have the following result:

$$
\begin{gathered}
\text { If }=\sum_{i=1}^{n} u_{i} f\left(x_{i}\right)+\sum_{i=1}^{n+1} v_{i} f\left(y_{i}\right)+d_{n \mu} c_{n \mu} M_{p_{n}}(f), \\
I f=\sum_{i=1}^{n+1} \bar{u}_{i} f\left(\bar{x}_{i}\right)+\sum_{i=1}^{n} \bar{v}_{i} f\left(\bar{y}_{i}\right)+d_{n-1, \mu+1} \bar{c}_{n \mu} M_{q_{n}}(f),
\end{gathered}
$$

where $d_{n \mu}$ is easily computable and does not vanish for $0<\mu \leqslant 2, \mu \neq 1$, and all $n \geqslant 2$. For $\mu=2$ we have the explicit expression

$$
d_{n \mu}= \begin{cases}\frac{2}{n+3}\left(\frac{n+1}{n+3}\right)^{m}, & n \text { even } \\ 4(n+2)(n+1)^{m-1} /(n+3)^{m+1}, & n \text { odd }\end{cases}
$$

where $m=[(n+1) / 2]$.

2. The Szegö Polynomials $E_{n+1, \mu}$. We give here the main results of Szegö with some minor modification of his notation and refer to [16] for details. See also Davis and Rabinowitz [3, pp, 82-89] with Monegato [11].

The Gegenbauer function of the second kind, $Q_{n}^{\mu}(z)$, defined by

$$
\begin{aligned}
Q_{n}^{\mu}(z) & =\frac{\Gamma(2 \mu)}{2 \Gamma(\mu+1 / 2)} \int_{-1}^{1} w(t ; \mu) \frac{C_{n}^{\mu}(t)}{z-t} d t \\
& =\frac{\Gamma(2 \mu)}{2 \Gamma(\mu+1 / 2)} z^{-n-1} \sum_{i=0}^{\infty} \beta_{i} z^{-2 i},
\end{aligned}
$$

where

$$
\beta_{i}=\int_{-1}^{1} w(t ; \mu) C_{n}^{\mu}(t) t^{n+2 i} d t, \quad i=0,1, \ldots,
$$

is analytic in the entire complex plane with a slit on the closed interval $[-1,1]$.

Hence

$$
\frac{1}{Q_{n}^{\mu}(z)}=z^{n+1} \sum_{i=0}^{\infty} \gamma_{i} z^{-2 i}=E_{n+1, \mu}(z)+\delta_{1} z^{-1}+\delta_{2} z^{-2}+\ldots,
$$

defining the polynomial $E_{n+1, \mu}(z)$ which is even (odd) for $n$ odd (even). Thus,

$$
Q_{n}^{\mu}(z) E_{n+1, \mu}(z)=1+b_{1} z^{-n-2}+b_{2} z^{-n-3}+\ldots,
$$

and by the argument given in [16] or [3]

$$
Q_{n}^{\mu}(z) E_{n+1, \mu}(z)=1+\sum_{i=0}^{n} c_{i} Q_{n+1+i}^{\mu}(z),
$$

for certain constants $c_{0}, \ldots, c_{n}$ depending on $\mu$ and $n$. Since $Q_{n}^{\mu}(z)$ is an odd (even) 
function if $n$ is even (odd), we have that $Q_{n}^{\mu}(z) E_{n+1, \mu}(z)$ is always an odd function which implies that $c_{0}=0$ if $n$ is odd.

Now, the functions of the second kind satisfy the following relations:

$$
\lim _{\epsilon \rightarrow 0}\left(Q_{n}^{\mu}(x+i \epsilon)-Q_{n}^{\mu}(x-i \epsilon)\right)=-i \pi \frac{\Gamma(2 \mu)}{\Gamma(\mu+1 / 2)} w(x ; \mu) C_{n}^{\mu}(x),
$$

$$
\lim _{\epsilon \rightarrow 0}\left(Q_{n}^{\mu}(x+i \epsilon)+Q_{n}^{\mu}(x-i \epsilon)\right)=2 \widetilde{Q}_{n}^{\mu}(x),
$$

where $\widetilde{Q}_{n}^{\mu}(x)$ is defined on the segment $[-1,1]$. Hence

$$
C_{n}^{\mu}(x) E_{n+1, \mu}(x)=\sum_{i=0}^{n} c_{i} C_{n+1+i}^{\mu}(x)
$$

and

$$
\widetilde{Q}_{n}^{\mu}(x) E_{n+1, \mu}(x)=1+\sum_{i=0}^{n} c_{i} \widetilde{Q}_{n+1+i}^{\mu}(x) .
$$

From (20) it follows that

$$
\int_{-1}^{1} w(x ; \mu) C_{n}^{\mu}(x) E_{n+1, \mu}(x) x^{k} d x=0, \quad k=0,1,2, \ldots, n,
$$

so that, by the theorem in [3, p. 77], an interpolatory integration rule based on the zeros of $C_{n}^{\mu}(x)$ and $E_{n+1, \mu}(x)$ is exact for all polynomials of degree $\leqslant 3 n+1$ which forms the basis for KEGGIR's.

Now, it can be shown that

$$
\begin{aligned}
Q_{n}^{\mu}(z) & =\gamma_{n \mu} w^{-n-1} F\left(1-\mu, n+1 ; n+\mu+1 ; w^{-2}\right) \\
& =\gamma_{n \mu} \sum_{j=0}^{\infty} f_{j \mu} w^{-n-1-2 j}
\end{aligned}
$$

where $z=1 / 2\left(w+w^{-1}\right), \gamma_{n \mu}=\sqrt{\pi} \Gamma(n+2 \mu) / \Gamma(n+\mu+1), F(a, b ; c ; z)$ is the usual hypergeometric function, $f_{0 \mu}=1$,

$$
f_{j \mu}=(1-\mu / j)(1-\mu /(n+\mu+j)) f_{j-1, \mu},
$$

and we have not shown the dependence on $n$ of the $f_{j \mu}$.

Setting $w=e^{-i \theta}$ and $x=\cos \theta$, we get that

$$
\widetilde{Q}_{n}^{\mu}(x)=\gamma_{n \mu} \sum_{j=0}^{\infty} f_{j \mu} T_{n+1+2 j}(x) .
$$

Since $E_{n+1, \mu}(x)$ contains only even or odd powers of $x$, we can write $E_{n+1, \mu}(x)$ in the form

$$
E_{n+1, \mu}(x)=\sum_{i=0}^{m-1} \lambda_{i \mu} T_{n+1-2 i}(x)+ \begin{cases}\lambda_{m \mu} T_{1}(x), & n \text { even }, \\ \frac{1}{2} \lambda_{m \mu}, & n \text { odd }\end{cases}
$$

where $m=[(n+1) / 2]$.

To determine the coefficients $\lambda_{i \mu}$, we equate, in view of (21) and (25), the coefficients of $T_{k}(x), k=1, \ldots, n+1$, in the product 


$$
\widetilde{Q}_{n}^{\mu}(x) E_{n+1, \mu}(x)=\gamma_{n \mu}\left(\sum_{j=0}^{\infty} f_{j \mu} T_{n+1+2 j}(x)\right)\left(\sum_{i=0}^{m}{ }^{\prime} \lambda_{i \mu} T_{n+1-2 i}(x)\right)
$$

to zero and the coefficient of $T_{0}(x)$ to unity. Here the prime means that if $n$ is odd, we replace $\lambda_{m \mu}$ by $1 / 2 \lambda_{m \mu}$. Since $T_{r}(x) T_{s}(x)=1 / 2\left(T_{r+s}(x)+T_{|r-s|}(x)\right)$, we see that the $\lambda_{i \mu}$ must satisfy the following equations

$$
\lambda_{0 \mu}=2 \gamma_{n \mu}^{-1}, \quad \sum_{i=0}^{k} f_{i, \mu} \lambda_{k-i, \mu}=0, \quad k=1, \ldots, m .
$$

Following Monegato [11], we define $\alpha_{i \mu}=\lambda_{i \mu} / \lambda_{0 \mu}$ so that $\alpha_{0 \mu}=1, \alpha_{1 \mu}=-f_{1 \mu}$, and

$$
\alpha_{k \mu}=-f_{k \mu}-\sum_{i=1}^{k-1} f_{i \mu} \alpha_{k-i \mu}, \quad k=2, \ldots, m .
$$

From this, we see that the $\alpha_{i \mu}$ are the first $m+1$ coefficients in the series

$$
\sum_{i=0}^{\infty} \alpha_{i \mu} u^{i}=\left\{\sum_{j=0}^{\infty} f_{j \mu} u^{j}\right\}^{-1}
$$

so that we can also use (29) for indices $k>m$. Here also we have not indicated the dependence on $n$ of the $\lambda_{i \mu}$ and $\alpha_{i \mu}$.

3. The Exact Degree of Precision of KEGGIR's and KELGIR's. Let us define

$$
f_{k}(x)=C_{n}^{\mu}(x) E_{n+1, \mu}(x) C_{n+1+k}^{\mu}(x), \quad k=0, \ldots, n .
$$

Then from (20) it follows that $I f_{k}=c_{k} h_{n+1+k, \mu}$. Since the KEGGIR applied to $f_{k}(x)$ vanishes, we have from (8) that $E_{p_{n}}\left(f_{k}\right)=c_{k} h_{n+1+k, \mu}$ so that the exact precision of the KEGGIR is determined by the first index $k$, say $k_{0}$, for which $c_{k_{0}} \neq 0$. Indeed, $p_{n}=3 n+1+k_{0}$. We now show that for $0<\mu \leqslant 2, \mu \neq 1, c_{0} \neq 0$ for $n$ even and $c_{1} \neq 0$ for $n$ odd.

Consider first the case $n$ even. Substituting (25) and (27) into (21) and equating the coefficients of $T_{n+2}(x)$, we find that

$$
\begin{aligned}
c_{0} \gamma_{n+1, \mu} & =\frac{\gamma_{n \mu}}{2}\left\{\lambda_{m \mu} f_{0 \mu}+\lambda_{m \mu} f_{1 \mu}+\lambda_{m-1, \mu} f_{2 \mu}+\cdots+\lambda_{0 \mu} f_{m+1, \mu}\right\} \\
& =\alpha_{m \mu}+\alpha_{m \mu} f_{1 \mu}+\alpha_{m-1, \mu} f_{2 \mu}+\cdots+\alpha_{1 \mu} f_{m \mu}+f_{m+1, \mu} \\
& =\alpha_{m \mu}-\alpha_{m+1, \mu}
\end{aligned}
$$

Thus, it suffices to show that $\alpha_{m \mu}-\alpha_{m+1, \mu}$ does not vanish. In fact, we shall show that the $\alpha_{i \mu}$ are strictly monotonic. For $0<\mu<1$, the sequence $\left\{f_{j \mu}\right\}$ is completely monotonic, i.e., $(-1)^{k} \Delta^{k} f_{j \mu}>0$ for all $j$ and $k[17$, p. 137]. Hence, by a theorem of Kaluza [6], the sequence $\left\{-\alpha_{i+1, \mu}\right\}$ is also completely monotonic and hence strictly monotonic. For $1<\mu<2$, the sequence $\left\{-f_{j+1, \mu}\right\}$ is completely monotonic. From this it follows, by some results in [6], that

$$
\frac{\alpha_{i-1, \mu}}{\alpha_{i \mu}}>\frac{\alpha_{i \mu}}{\alpha_{i+1, \mu}}, \quad i=1,2, \ldots
$$


Since $\Sigma_{i=0}^{\infty} \alpha_{i \mu}$ converges, and in fact equals $\{F(1-\mu, n+1 ; n+\mu+1 ; 1)\}^{-1}$, it follows that the sequence $\left\{\alpha_{i \mu}\right\}$ is strictly monotonic. For $\mu=2$, Szegö [16] gives an explicit expression for the $\lambda_{i \mu}$,

$$
\lambda_{i 2}=\frac{2}{\sqrt{\pi}} \frac{1}{n+3}\left(\frac{n+1}{n+3}\right)^{i}, \quad i=0,1, \ldots,
$$

which again shows that the $\alpha_{i 2}$ are strictly monotonic.

We now consider the case $n$ odd. Proceeding as before, this time equating the coefficients of $T_{n+3}(x)$, we find that

$$
\begin{aligned}
c_{1} \gamma_{n+2, \mu}= & \frac{\gamma_{n \mu}}{2}\left\{\lambda_{m \mu} f_{1 \mu}+\lambda_{m-1, \mu} f_{0 \mu}\right. \\
+ & \lambda_{m-1, \mu} f_{2 \mu} \\
& \left.+\lambda_{m-2, \mu} f_{3 \mu}+\cdots+\lambda_{0 \mu} f_{m+1, \mu}\right\} \\
= & \alpha_{m-1, \mu}+\alpha_{m \mu} f_{1 \mu}+\alpha_{m-1, \mu} f_{2 \mu}+\cdots+\alpha_{1 \mu} f_{m \mu}+f_{m+1, \mu} \\
= & \alpha_{m-1, \mu}-\alpha_{m+1, \mu} .
\end{aligned}
$$

Since the $\alpha_{i \mu}$ are strictly monotonic, it follows that $c_{1} \neq 0$.

For $\mu=0, f_{j 0}=1, j=0,1,2, \ldots$, so that $\lambda_{00}=-\lambda_{10}=2 n / \pi^{1 / 2}, \lambda_{i 0}=0$,

$i>1$ and $E_{n+1,0}=\left(2 n / \pi^{1 / 2}\right)\left\{T_{n+1}(x)-T_{n-1}(x)\right\}, n \geqslant 2$. Hence

$$
\begin{aligned}
C_{n}^{0}(x) E_{n+1,0}(x) & =k_{1} T_{n}\left\{T_{n+1}-T_{n-1}\right\}=\frac{k_{1}}{2}\left\{T_{2 n+1}-T_{2 n-1}\right\} \\
& =k_{2}\left(1-x^{2}\right) U_{2 n-1}=k_{3}\left(1-x^{2}\right) C_{2 n-1}^{1}(x),
\end{aligned}
$$

and the zeros of $C_{n}^{0}(x) E_{n+1,0}(x)$ are the abscissas of the $(2 n+1)$-point LGIR for the weight $w(x ; 0)$ which is of exact precision $4 n-1$, as can also be seen from the fact that $c_{n-2}$ is the first $c_{k}$ which does not vanish.

For $\mu=1, f_{01}=1, f_{j 1}=0, j>0$ so that, $\lambda_{01}=2 / \sqrt{\pi}, \lambda_{i 1}=0, i>0$, and $E_{n+1,1}(x)=(2 / \sqrt{\pi}) T_{n+1}(x)$. Hence

$$
C_{n}^{1}(x) E_{n+1,1}(x)=k_{1}^{\prime} U_{n}(x) T_{n+1}(x)=k_{2}^{\prime} C_{2 n+1}^{1}(x),
$$

and the zeros of $C_{n}^{1}(x) E_{n+1,1}(x)$ are the abscissas of the $(2 n+1)$-point GGIR for the weight $w(x ; 1)$ which is of exact precision $4 n+1$ and which also follows from the fact that $c_{n}$ is the first $c_{k}$ which does not vanish.

In the case of the KELGIR, we define

$$
\bar{f}_{k}(x)=\left(1-x^{2}\right) C_{n-1}^{\mu+1}(x) E_{n, \mu+1}(x) C_{n+k}^{\mu+1}(x), \quad k=0,1, \ldots, n-1,
$$

so that $I \bar{f}_{k}=c_{k} h_{n+k, \mu+1}$. Hence, since $c_{0}=c_{0}(n-1, \mu+1) \neq 0$ for $n-1$ even, i.e., for $n$ odd, while $c_{1} \neq 0$ for $n-1$ odd, we have that the $(2 n+1)$-point KELGIR is of exact precision $3 n+1$, for $n$ even, and $3 n$, for $n$ odd, provided that $\mu \neq 0$. For $\mu=0$, we have as before that $E_{n 1}(x)=\left(2 / \pi^{1 / 2}\right) T_{n}(x)$, so that

$$
\left(1-x^{2}\right) C_{n-1}^{1}(x) E_{n 1}(x)=\hat{k}_{1}\left(1-x^{2}\right) C_{2 n-1}^{1}(x),
$$

whose zeros are again the abscissas of the $(2 n+1)$-point LGIR for the weight $w(x ; 0)$. 
If we now define

$$
d_{n \mu}= \begin{cases}\alpha_{m \mu}-\alpha_{m+1, \mu}, & n \text { even, } \\ \alpha_{m-1, \mu}-\alpha_{m+1, \mu}, & n \text { odd, } m=[(n+1) / 2],\end{cases}
$$

we have that for the Gauss case

$$
d_{n \mu}= \begin{cases}c_{0} \gamma_{n+1, \mu}, & n \text { even } \\ c_{1} \gamma_{n+2, \mu}, & n \text { odd }\end{cases}
$$

while for the Lobatto case

$$
d_{n-1, \mu+1}= \begin{cases}c_{0} \gamma_{n, \mu+1}, & n \text { even, } \\ c_{1} \gamma_{n+1, \mu+1}, & n \text { odd }\end{cases}
$$

where we have suppressed the dependence of $c_{0}$ and $c_{1}$ on $n$ and $\mu$. This leads us immediately to formulas (10) and (11). For example, applying (8) with $n$ even to $f_{0}(x)$, we have that

$$
c_{0} h_{n+1, \mu}=K_{n \mu} k_{n \mu} 2 \gamma_{n \mu}^{-1} 2^{n} k_{n+1, \mu}(3 n+2) !,
$$

so that

$$
K_{n \mu}=\frac{d_{n \mu}}{\gamma_{n+1, \mu}} \frac{h_{n+1, \mu} \gamma_{n \mu}}{2^{n+1} k_{n \mu} k_{n+1, \mu}(3 n+2) !}=\frac{d_{n \mu} c_{n \mu}}{2^{p_{n} p_{n} !}}
$$

For $n$ odd, we consider $f_{1}(x)$ while in the Lobatto case we work with $\bar{f}_{0}(x)$ and $\bar{f}_{1}(x)$.

4. Remarks. a. Monegato [11] gives an error bound for KEGGIR's with $0<$ $\mu<1$. We shall show how to improve this bound slightly and extend it to the case $1<\mu<2$, as well as to KELGIR's with $-1 / 2<\mu \leqslant 1, \mu \neq 0$.

For $n$ even, Monegato writes the error $E_{p_{n}}(f)$ for $f \in C^{3 n+2}[-1,1]$ in the form

$$
E_{p_{n}}(f)=\frac{2^{-2 n}}{k_{n \mu}(3 n+2) !} \int_{-1}^{1} w(x ; \mu) C_{n}^{\mu}(x)\left(\bar{E}_{n+1, \mu}(x)\right)^{2} f^{(3 n+2)}\left(\xi_{x}\right) d x
$$

where

$$
\bar{E}_{n+1, \mu}(x)=E_{n+1, \mu}(x) / \lambda_{0 \mu}=\sum_{i=0}^{m} \alpha_{i \mu} T_{n+1-2 i}(x)
$$

Hence

$$
\left|E_{p_{n}}(f)\right| \leqslant \frac{\pi \Gamma(n+2 \mu) B_{n+1, \mu}^{2}}{2^{3 n+2 \mu-1} p_{n} ! \Gamma(\mu+1) \Gamma(n+\mu)} M_{p_{n}}
$$

where

$$
M_{s}=\max _{-1 \leqslant x \leqslant 1}\left|f^{(s)}(x)\right| \text { and } \quad B_{n+1, \mu}=\max _{-1 \leqslant x \leqslant 1}\left|\bar{E}_{n+1, \mu}(x)\right|
$$


For $0<\mu<1$, Monegato states that $B_{n+1, \mu}<2$ and replaces $B_{n+1, \mu}$ by 2 in (45). Now, while this bound is the best available for $0<\mu \leqslant 1 / 2$, we can improve on it for $1 / 2<\mu<1$. In addition, a bound on $B_{n+1, \mu}$ is also available for $1<\mu \leqslant 2$. This follows from our observation above that

$$
\begin{aligned}
\sum_{i=0}^{\infty} \alpha_{i \mu} & =\{F(1-\mu, n+1 ; n+\mu+1,1)\}^{-1} \equiv T_{n \mu} \\
& =\frac{\Gamma(\mu) \Gamma(n+2 \mu)}{\Gamma(n+\mu+1) \Gamma(2 \mu-1)}, \quad \mu>1 / 2, \mu \neq 1,2 .
\end{aligned}
$$

Now for $1 / 2<\mu<1, \alpha_{0 \mu}=1, \alpha_{i \mu}<0, i>0$. Since

$$
B_{n+1, \mu} \leqslant \sum_{i=0}^{m}\left|\alpha_{i \mu}\right|=1-\sum_{i=1}^{m} \alpha_{i \mu}<1-\sum_{i=1}^{\infty} \alpha_{i \mu} \text {, }
$$

it follows that $B_{n+1, \mu}<2-T_{n \mu}<2$. For $1<\mu<2$, we have that $\alpha_{i \mu}>0$, for all $i$. Hence $B_{n+1, \mu} \leqslant \sum_{i=0}^{m} \alpha_{i \mu}<T_{n \mu}$. For $\mu=2$,

$$
\sum_{i=0}^{\infty} \alpha_{i 2}=\left(1-\frac{n+1}{n+3}\right)^{-1}=\frac{n+3}{2}>B_{n+1,2}
$$

For $n$ odd, using classical arguments, we have the same bound.

In the Lobatto case, we have, similarly for $n$ odd, that

$$
E_{q_{n}}(x)=\frac{2^{2-2 n}}{k_{n-1, \mu+1}(3 n+1) !} \int_{-1}^{1} w(x ; \mu+1) C_{n-1}^{\mu+1}(x)\left(\bar{E}_{n, \mu+1}(x)\right)^{2} f^{(3 n+1)}\left(\bar{\xi}_{x}\right) d x
$$

whence

$$
\left|E_{q_{n}}(f)\right| \leqslant \frac{\pi \Gamma(n+2 \mu+1) B_{n, \mu+1}^{2}}{2^{3 n+2 \mu-2} q_{n} ! \Gamma(n+\mu) \Gamma(\mu+2)} M_{q_{n}},
$$

where for $-1 / 2<\mu<0, B_{n, \mu+1}<2-T_{n-1, \mu+1}$ and for $0<\mu<1, B_{n, \mu+1}<T_{n-1, \mu+1}$. For $\mu=1, B_{n 2}<(n+2) / 2$. As before, the same bound holds for $n$ even.

b. The Fourier-Gegenbauer coefficients of a function $f(x)$ are defined by

$$
\mathrm{FG}_{n \mu}(f)=h_{n \mu}^{-1} \int_{-1}^{1} w(x ; \mu) C_{n}^{\mu}(x) f(x) d x, \quad n=0,1, \ldots
$$

As Barrucand [2] points out, the integral is most efficiently evaluated by a $(2 n+1)$ point KEGGIR applied to the function $C_{n}^{\mu}(x) f(x)$ which reduces to the $(n+1)$-point formula

$$
\mathrm{FG}_{n \mu}(f) \simeq h_{n \mu}^{-1} \sum_{i=1}^{n+1} v_{i} C_{n}^{\mu}\left(y_{i}\right) f\left(y_{i}\right)=\sum_{i=1}^{n+1} \tilde{v}_{i} f\left(y_{i}\right) .
$$

For $\mu \neq 0,1$, we get a rule which is exact for polynomials of degree $<p_{n}-n$, which is the best possible. For assume that there existed an $(n+1)$-point rule, say

$$
\mathrm{FG}_{n}(f) \simeq \sum_{i=1}^{n+1} \hat{v}_{i} f\left(\hat{y}_{i}\right),
$$

exact for polynomials of degree $p_{n}-n, n$ even. This would imply that 


$$
\int_{-1}^{1} w(x ; \mu) C_{n}^{\mu}(x) E_{n+1, \mu}(x) \prod_{i=1}^{n+1}\left(x-\hat{y}_{i}\right) d x=0,
$$

which contradicts our results above. Similarly for $n$ odd.

For $\mu=0$, the rule (50) is exact for polynomials of degree $\leqslant 3 n-1$, a result which has already been reported in [8]. For $\mu=1,(50)$ is exact for polynomials of degree $\leqslant 3 n+1$ which is the best possible result, so that the highest precision is achieved for Fourier-Chebyshev coefficients of the second kind. However, we should warn the user that the weights $\tilde{v}_{i}$ in (50) alternate in sign inasmuch as the $v_{i}$ are positive and the zeros of $C_{n}^{\mu}(x)$ separate those of $E_{n+1, \mu}(x)$, so that the $C_{n}^{\mu}\left(y_{i}\right)$ alternate in sign.

Department of Applied Mathematics

The Weizmann Institute of Science

Rehovot, Israel

1. P. BARATElla, "Un'estensione ottimale della formula di quadratura di Radau," Rend. Sem. Mat. Univ. Politec. Torino, v. 37, 1979, pp. 147-158.

2. P. BARRUCAND, "Intégration numérique, abscisse de Kronrod-Patterson et polynômes de Szegö," C. R. Acad. Sci. Paris Sér. A, v. 270, 1970, pp. 336-338.

3. P. J. DAVIS \& P. RABINOWITZ, Methods of Numerical Integration, Academic Press, New York, 1975.

4. A. ERDÉLYI, W. MAGNUS, F. OBERHETTINGER \& F. G. TRICOMI, Higher Transcendental Functions, Vol. II, McGraw-Hill, New York, 1953.

5. D. K. KAHANER \& G. MONEGATO, "Nonexistence of extented Gauss-Laguerre and Gauss-Hermite quadrature rules with positive weights," Z. Angew. Math. Phys., v. 29, 1978, pp. 983-986.

6. T. KALUZA, "Über die Koeffizienten reziproker Potenzreihen," Math. Z., v. 28, 1928, pp. $161-170$.

7. A. S. KRONROD, Nodes and Weights for Quadrature Formulae. Sixteen Place Tables, "Nauka", Moscow, 1964; English transl., Consultants Bureau, New York, 1965.

8. C. A. MICCHELLI \& T. J. RIVLIN, "Turán formulae, and highest precision quadrature rules for Chebyshev coefficients," IBM J. Res. Develop., v. 16, 1972, pp. 372-379.

9. G. MONEGATO, "A note on extended Gaussian quadrature rules," Math. Comp., v. 30, 1976, pp. 812-817.

10. G. MONEGATO, "Positivity of the weights of extended Gauss-Legendre quadrature rules," Math. Comp., v. 32, 1978, pp. 243-245.

11. G. MONEGATO, "Some remarks on the construction of extended Gaussian quadrature rules," Math. Comp., v. 32, 1978, pp. 247-252.

12. G. MONEGATO, "An overview of results and questions related to Kronrod schemes," in Numerische Integration (G. Hämmerlin, Ed.), ISNM 45, Birkhäuser Verlag, Basel, 1979, pp. 231 240.

13. T. N. L. PATTERSON, "The optimum addition of points to quadrature formulae," Math. Comp., v. 22, 1967, pp. 847-856.

14. R. PIESSENS \& M. BRANDERS, "A note on the optimal addition of abscissas to quadrature formulas of Gauss and Lobatto ty pe," Math. Comp., v. 28, 1974, pp. 135-139.

15. JU. S. RAMSKIĬ, "The improvement of a certain quadrature formula of Gauss type," Vyčisl. Prikl. Mat. (Kiev), v. 22, 1974, pp. 143-146. (Russian)

16. G. SZEGÖ, "Über gewisse orthogonale Polynome, die zu einer oszillierenden Belegungsfunktion gehören,” Math. Ann., v. 110, 1934, pp. 501-513.

17. G. SZEGÖ, Orthogonal Polynomials, 4th ed., Amer. Math. Soc. Colloq. Publ., vol. 23, Amer. Math. Soc., Providence, R.I., 1975; reprinted 1978. 